\title{
Comparing Australian my health record system implementation With global best practices with recommendations
}

\author{
Sultan Sulaiman Alharbi * \\ Prince Sultan Military Medical City, Riyadh, Saudi Arabia
}

\author{
Key Words: \\ Australia \\ Healthcare \\ Health science \\ Digital health \\ Recommendation
}

Received: 14 December 2016

Accepted: 24 April 2017

Published: 30 June 2017

\begin{abstract}
An Electronic Health Record (EHR) system is a computerized medical information system that collects, displays, and stores a patient's information. It is an evidence base that addresses issues associated with patients' paper records. Implementing such a system will have a high positive impact on healthcare quality and healthcare services. For example, an EHR is an electronic record that sequentially stores any resident's health data from nearly the first month of gestation until death and can bring those records anytime and to any authorized physician. This study aims to investigate the present status of EHR implementations around the world and identify best practice solutions. Additionally, the study focuses on how to adopt best practices in Australia. The methodology of this paper involved academic research consisting of 250 articles and over 100 websites. This paper's information was obtained through a search strategy-using PubMed, Google Scholar, and Google - of the best practices applied in many countries, including the US, Canada, and several nations in Western Europe. With 30 references, the recommendations were provided to adopt the best practice solutions for the Australian My Health Record system while implementation. This paper has further exposed the problems with EHR systems as implemented worldwide. The recommendations can be summarised as follows: improve the overall awareness of the stakeholders, conduct training sessions for stakeholders on the My Health Record system, reward physicians for using the system, achieve ongoing technical and systems security integrity and compliance, implement a response plan in the event of a breach of the EHR system, and implement a simple graphical user interface to facilitate access to stakeholders. Further results as recommendations are provided in the results section. The research concluded that Australia would achieve a stable healthcare system by adopting these best practice solutions, which will ensure a higher level of healthcare quality to patients and healthcare alike. This paper will give stakeholders a clear vision to determine the original cause that hinders satisfying results while implementing the My Health Record system in Australia.
\end{abstract}

(C) 2017 The Author(s). Published by TAF Publishing.

\section{INTRODUCTION}

An EHR system is a computerised medical information system that collects, displays, and stores patients' information [1]. According to Health IT [2], an EHR is a patient's paper records and information in digital format, available directly and securely to any authorised users. EHR systems have been the subject of a number of substantial deals in developed countries, as have healthcare providers. Although to date, they are not used extensively, these systems are expected to provide significant savings in healthcare, enhance healthcare quality, and reduce health errors [3]. The key concept of an EHR system is to function

\footnotetext{
* Corresponding author: Sultan Sulaiman Alharbi

†Email: ssn.harbi@gmail.com
} 
as a collection of constantly updated information related to a patient's health. The EHR system can be an effective electronic record that continually stores a resident's medical data from nearly the first month of gestation until death. EHR systems are built to ensure all of a patient's information is available to other healthcare providers and organisations [4]. An electronic health record can include a patient's medical account, identity, treatment schedule, immunisation, allergies, test outcomes, as well as immunisation dates [5].

EHR systems also computerise and rationalise the healthcare provider's workflow. It is clear that one of the main characteristics of an EHR is that it allows health data to be formulated and handled by sanctioned providers in a digital design capable of being shared with various external entities providing support to the primary healthcare organisation. For example, in order to provide appropriate services, laboratories, specialists, hospital admissions departments, doctors, pharmacies, and emergency facilities each require information from the clinician involved in a patient's care [6]. The medical and treatment histories represent the entirety of the primary organisational clinical information collected for patients. EHR is a system aimed at bringing about significant improvements in care delivery among organisations with high quality of patient care with acceptable outcomes [7]. Thus, this information is considered to be appropriate to that individual's care under any particular provider. The broader definition of EHR can be seen as a digital report of a patient's paper chart [6].

Moreover, EHR systems are a new version of patients' data, in digital record format, that can be stored and exchanged with several authorised users in a secure way [8].

\section{Study Purpose}

As this paper finds that My Health Record system needs more than 15 years to enrol all Australian citizens in the system. This research will investigate the current system in Australia, "My Health Record", to illustrate the barriers that have prevented successful implementation. In addition, the aim of this paper is to investigate and analyse the best practice solutions adopted in EHR systems that have been implemented around the world. Following this, recommendations will involve encouraging stakeholders to participate in a successful system implementation, as well as strategies to overcome the problems other countries have experienced in the implementation of EHR systems.

\section{LITERATURE REVIEW}

\section{Historical Perspective of Electronic Health Record Systems}

The first application related to medicine was found by Herman Hollerith [9]. It was based on the punched card data processing technique and was created for the 1890 census in America. The methods in his technique were adapted to epidemiologic and health surveys [9]. The same technique was then adopted in the development of hospital systems in 1920s, 1930s, and 1940s [9]. After that, the first modern digital computer systems were devolved in America during the Second World War. In 1946 came the first release of ENIAC, and it became common in the marketplace in 1950s $[9,10]$. Ledley and Lusted in the 1950's recognized that computers could be useful for medical diagnosis and treatment. They reasoned these because computers could process and archive information more rapidly than humans $[10,11,12]$.

Dr. Barnett started working for hospital information system in 1960s and created many hospital application programs for about thirty years [9]. In 1962 BBN, a firm in Cambridge named Bolt Beranek and Newman established a time-sharing technology that had online database. BBN had a vision of this system which would have a beneficial impact on 
information processing needed in hospitals [13]. Then during 1964 to 1966, G. Octo developed medications ordering system, lab reporting system, and admission discharge census system. The main development principle being used in the lab by that time was "modular approach" [13]. The development of Hospital Information Systems (HIS) increased in 1970s. For supporting all the applications, one efficient computer was required which could run the integrated designing of a time-shared computer, a large and single computer would be used to support a collection of applications. In 1970s, another technology of "biomedical computing activity" was introduced [9]. The standardized facilities to the individuals and less time training were provided by the general purpose software tools [9]. Today, the healthcare practitioners can install the variety of applications related to clinical research and patient care in their personal computers that can easily be accessed. In the article "HISTORY OF MEDICINE; Development of the Electronic Health Record", Jim Atherton described the history of development of The Healthcare Information and Management Systems Society (HIMSS).

He published his paper in "American Medical Association Journal of Ethics" in March 2011. In 1968, G. Octo Burnett put forth his research about the historical perspective of EHR system in his article, "History of development of medical information systems" at the laboratory of computer science at Massachusetts General Hospital [13]. According to this article, the actual development of electronic health record system was started in 1960s and 1970s [14]. In that era, the health centers started developing their own health record systems. The very first and most efficient electronic health record system present at that time was called a clinical information system. Then a clinical decision support system, called a "Health Evaluation through Logical Processing" was created by 3M and the University of Utah [14]. In 1968, at the Massachusetts General Hospital, the Computer Stored Ambulatory Record system was implemented. In the 1970s, the use of "Decentralized Hospital Computer Program" was started by the federal government [14]. By that time, the medical students and the healthcare practitioners used to use "Computerized Patient Record System". In these systems' databases, some features like recognized several terms of the same disease.

This feature allowed patients to recognize any condition across the health system notwithstanding the differences in terminology at different organizations [14]. Then further investigations and research were done to add to the existing properties of EHR. The electronic health record system was incorporated in American Recovery and Reinvestment Act of 2009 by President Obama as a part of the Health Information Technology for Economic and Clinical Health Act [14]. The electronic technologies became more attractive and affordable for the implementation in the healthcare systems due to their ability to access and store information.

\section{The Development of the EHR}

Diverse kinds of EHR have been developed by several administrations, and hospitals with the objective of assembling the patient's information so that they can be viewed readily and administered at one place. It is clear that the development of EHR can be partitioned into two primary periods. In the early 1980's, stakeholders observed the gains (fast process, data availability) of health systems and started creating information systems that could handle the wider problems that could help in the general use of the electronic health formation [9].

The earliest EHR systems employed the title of clinical information systems. Around the mid-60s, Lockheed created a HIS, which was later presented to the Technicon Data 
Systems (TDS), and after that, this system was a part of Eclipsys, Inc [9]. At that time, there was a collaboration between the University of Utah and 3M to develop the earliest clinical decision support system, which is Health Evaluation through Logical Processing (HELP) [9]. HELP was a system used by hospitals to aid decision-making that also provided reminders and alerts for upcoming appointments. It was revolutionary back then. In 1968, the Computer Stored Ambulatory Record (COSTAR) started at Massachusetts General Hospital [14]. COSTAR was an EHR system available to societies via public domainsupported product [13]. It was formulated in partnership with Harvard; thus, it was seen that COSTAR contained some new features compared to the HELP system like automated appointment scheduling, clinical reminders, a tumor registry, storage, and retrieval of data. It also provided clinical reporting and patient record inquiry [13].

Since the 1980's, more concentrated attempts have been made to augment the use of EHR. In the 1980's, the Institute of Medicine (IOM) announced their needs to evaluate the paper health records [14]. In 1991, they published their reported results and in 1997, there was a 2nd edition of their report containing recommendations to improve patients' records. The published results indicated that by employing the EHR, patient's records were much safer as compared to the manual way of doing things like critical role of needs of instant information especially when doctors need decisions instantly [14]. The IOM, reported problems, and technical barriers like interface issues, text processing, and security. Moreover, the report mentioned there were some non-technological barriers related to missing data [13]. From 2000 until 2016, there were several of EHR systems that overcame most of the issues mentioned above. For instance OpenEHR. OpenEHR is a collection of open specifications for EHR architecture, but it is, however, not software. Its design purpose has been to allow the semantic interoperation of health information within and between EHR systems, avoiding vendor lock-in data. OpenEHR gained international momentum as it can be seen in high implementations of the OpenEHR specifications, for instance, as in the UK National Health Service program. The system is an intellectual property for OpenEHR Foundation, and it was created through the partnering of Ocean Informatics [15]. Leslie stated that though there have been many EHR models, OpenEHR has survived time because of many differentiating factors such as: Open source initiative OpenEHR is available freely to everyone under open licensing [15].

Language independence - Archetypes, which are used in OpenEHR, are independent of language. They can be created in one language and be translated in any language. As such, they are available in any country. The separation of the clinical and technical sectors The design of OpenEHR is such that its technical constituents can be separated from the dynamic clinical knowledge [16]. Easily implemented - There is little infrastructure required to implement OpenEHR due to the archetypes that can be separately developed from the software application and the use of the object-oriented model [16]. Knowledge-enabledcapturing - It captures the complex and dynamic state of health information. Medical practitioners can easily and actively contribute to the development of the clinical knowledge base through the archetypes. These archetypes can then be revised to reflect the changing clinical knowledge [16].

\section{RESEARCH METHODOLOGY}

\section{Problem Statement}

Australian health care providers have passed a long way towards effective implementation of EHR system. The first system that was called Personal Control Electronic Health Record System (PCEHR) was implemented in 2012. Regardless of the fact that the system 
was highly promising, the statistics were far from demonstrating success. According to the national data, approximately two million people have been enrolled into usage of the system for the period of two years. Such number of people was not acceptable neither for the government nor for the process of adaptation of the system, especially taking into account the fact that more than $\$ 699$ million were spent to support the initiative. In 2016, the government has made a decision to rename the PCEHR system to be My Health Record system. As the authorities and health care administration teams recognized it, the system is currently under the process of improvement as it still has numerous problems, including the one of enrolling more citizens. According to calculations in this paper, the system will need more than 15 years to enrol all citizens of Australia. Such situation cannot be considered effective neither for health care provision of the nation nor for the economy of the country. This means that urgent measures must be taken to improve the process of implementation of the system and facilitate the process of patient enrolment.

Thus, the main problem of the study is lack of knowledge of Australian EHR developers about innovative means of improvement of the system to enrol more citizens. Targeting the problem, the study tends to find the information on success of the system in other countries and also develop a strategy that would introduce the found chances to the Australian EHR system without any significant stress for health care practitioners, people who already use the system, and also those who only have such opportunity.

\section{Methodology Tools}

The problem of the current study and its aim have determined methodology applied to conduct the research. Since the two are about assessment of My Health Record system that is applied in Australia, implementation of qualitative research methods became the main means to identify challenges of the system, as well as its advantages and failures. Computer science vision of the problem and aim of the research have played a critical role in terms of the study conduction. Review of the recent literature that is related to the issue has been developed due to availability of reviews and records to be observed. This means that the study was carried out on the basis of analysis of documents that were chosen from hospitals and national EHR systems, demonstrating both failures and advantages of it. Based on successful enrolment of people and a broad experience, a higher level of objectivity requires random choosing of hospitals and national systems to study their EHR systems.

National EHR systems of the United States of America, Canada, and Europe were chosen due to their broad experience in terms of using EHR systems, as well as high level of development of the countries, which ensures the fact that their citizens are more likely to use EHR systems of the highest possible quality. Taking the above approach as grounds, the current paper investigates a range of criteria that are taken by the above-mentioned countries to make the EHR systems adapted to the needs of their patients and requirements of their health care practitioners to ensure successful enrolment. Discussion of the factors allows judging on success or failure of the systems and concluding about further actions to be taken to contribute to enrol citizens. The study involves progress covering of enrolling citizens in various countries as a factor that allows measuring advantages and disadvantages of the system, providing reasons for claiming them effectively or not. It is important to pay attention to the fact that methodology of the current study considers the high level of security, as well as opt level in to EHR system. Reaching objectivity in terms of results of the study requires considering not only countries that enrol all their citizens into usage of the system, but also the locations where EHR are still not popular. Moreover, the 
research focuses both on systems that are considered secure and advance, as well as on those requiring urgent improvement for safety of the patients. The research strategy used for the current study included several main stages. The first one was choosing a research topic, turning it into a question, and identifying keywords like EHR, Healthcare, Health science, Digital health, Recommendation. Gathering background information from reputable and reliable sources of information from PubMed, Google Scholar, and Google of the best practices that have been applied in the countries mentioned above. These included electronic books, journal articles, politician speeches, and websites. Evaluation and comparison were the next stages to identify usefulness of EHR systems in terms of improvement of the Australian one. Discussion and conclusion were the closing stages of the research strategy.

\section{RESULTS}

Opt Out

My Health Record system patients have the option to opt out of the system at any time. This creates a challenge, as only a fully accessible and fully inclusive health database is beneficial to the health sector. However, concerns about security make supporting a user opt out of the system unavoidable. Until security is sufficiently enhanced for patients to trust the system, this issue will continue to impede nationwide application [17]. To avoid this in the future, Australian citizens will automatically enrol in the My Health Record system, and there will be no choice to opt out of the system as it is considered as nationally beneficial [18].

\section{Reduce Risk and Separate System in Each State}

There are numerous ways to reduce the risk of sending patients' information to other patient records. These include using authentication, encryption, and user interface design [19]. Encryption can play an important part for making sure that any information that gets misplaced will not be seen by anyone else. Furthermore, setting up a separate system for each state will play a significant role in achieving full control. Decentralisation to state-run EHR systems allows each jurisdiction in Australian states to be responsible for selecting and obtaining their own health IT systems, as well as for monitoring the outputs of the system as in Sweden [18]. Thus, this will add significant value to efforts toward successful implementation similar to that achieved in Sweden.

\section{Financial Incentives and Rating}

Among the medical community, though the EHR would supposedly make doctors' work easier and more efficient with improved patient care, there has been considerable resistance to the new system. Among the reasons for this resistance is the fear of increased liability for healthcare practitioners with the use of the electronic health systems. Mistakes are bound to occur as the new system is installed and utilised for the first time, and many healthcare practitioners fear that these will form the basis for malpractice suits. The electronic time records in such a system would provide evidence against a physician in the event that notes are not recorded at the time of visit, thereby resulting in the illegal alteration of information.

Furthermore, among general practitioners, there is even poorer uptake of the system. These doctors often see the same patients repeatedly and may follow them all their lives. Thus, they have all the information that would be needed regarding the patients' past health, and see little or no benefit in the new system, but simply an additional work- 
load placed in their already busy schedules. This has raised challenges in implementing the system, as these general practitioners are the ones who hold most of the vital information regarding their patients' health that would be beneficial to another doctor treating the same patient at another location. This has led some governments to issue a financial incentive to doctors to encourage them to upload their patients' records onto the system [20].

There were studies conducted in the US to examine the efficiency of the EHR incentive plan which showed that EHR adoption has expanded dramatically since financial incentives started to be offered [21]. Therefore, a financial incentive may play a significant role in the successful implementation of the My Health Record system. In addition, rating those using or not using the new system may also make a difference, especially if the government announces this measure to the community. To illustrate, in Denmark, the government rates and gives GPs that use HER system a high rate and others will take a low rate to improve the implementation of EHR system [22, 23].

\section{DISCUSSION}

In Australian Context and based on recommendations from the National Health and Hospitals Reform Commission 2008 (NHHRC), the Australian government announced a national plan to start a PCEHR system. By 1 July 2012, the Australian government had officially launched this new system [24]. The Australian government invested $\$ 466$ million to commission the PCEHR in the 2010/2011 budget [25]. The PCEHR system was an "opt-in" system to capture health data electronically [26].

The government in May 2012 with an additional \$233.7 million further funded it. Nine implementation sites were funded by the government when the EHR pilot programme began in 2011. More than $\$ 55$ million was provided to take care of the patients and to register for the PCEHR [27]. The Australian Senate passed legislation in 2012 for the institutionalisation of PCEHR as a part of Australia's health system [27]. The programme was rolled out successfully and by mid-2013, had more than 520,000 registered patients and over 5,000 general practitioners [27]. The EHR system in Australia has been reliable and has provided a secure source of important clinical information, facilitating communication among practitioners. The EHR system has enabled various health practitioners to help patients receive healthcare and treatment through various available means. Access has also been provided to patients and professional healthcare providers to help them regulate their personal health records [28]. The Australian government further supported the PCEHR system with $\$ 140$ million, provided in the 2014/15 budget, to continue the roll-out of the system [29].

From July 2012 to 2014, PCEHR had 2.5 million registered patients and approximately 5,100 registered GPs [30]. However, it has not been efficient in its usability, governance, clinical utility, or operations [30]. In 2014, PCEHR was decommissioned from the Australian government due to the relatively low number of registrations; indeed, Ms Ley illustrated that less than 1 out of 10 Australians had signed up to the PCEHR system [31]. However, in 2016, the system was renamed to My Health Record with a rescue package of AUD 485 million to support the new system [32]. The EHR system in Australia strives to ensure that individual privacy is respected, announcing fines up to AUD 500,000 or a prison sentence, or both, for any individual who tries intentionally to obtain other users' data from health records, or does so through misuse of the system [32]. The automation of health records has facilitated interaction between patients and clinicians [32]. Adoption of EHR in Australia has: enabled the sharing of valuable information among healthcare 
professionals, increased efficiencies in the support of healthcare services, and reduced the number of hospitalisation cases. My Health Record is a digital platform created by the Australian government to provide patient information to healthcare providers through online channels. My Health Record provides most aspects of individuals' health information summaries, such as allergies, medical prescriptions, and reactions, if any. My Health Record was previously called the PCEHR system [33]. It allows various individual doctors, healthcare centres, and hospitals to access and view patients' health information, as permitted by the access control features [32]. Individuals can access their health records online whenever they want. In contrast with its previous version, My Health Record system is an online "opt-out" system in which individuals in Australia can register for the system and opt out of it online.

They are responsible for their health information, which they can communicate to healthcare professionals. The individuals have a choice to opt out of the system whenever they feel unsafe, and have the ability to delete their accounts and information [32]. My Health Record is regulated by the My Health Record Act, which stipulates how individuals' health information can be collected, how it will be used, as well as the rules of its disclosure. Therefore, any form of unauthorised collection, use, or disclosure of individual health information contained in My Health Record breaches the My Health Record Act, and concurrently interferes with an individual's privacy [32].

\section{Lack of Registration}

Statistics released by the Australian Digital Health Agency (Figure 1), on 21 August 2016, demonstrate the number of consumers who have registered for My Health Record. The data indicate that $4,079,339$ consumers-representing $17 \%$ of the Australian populationhave registered.

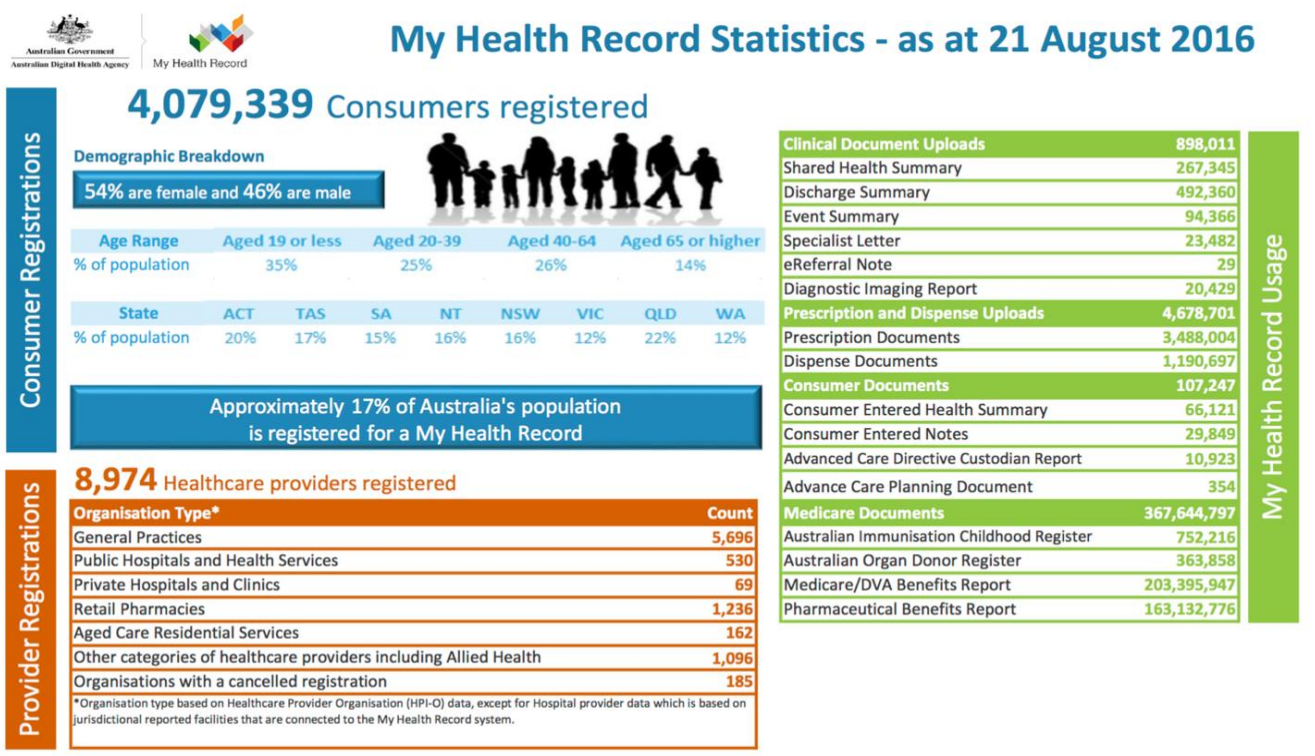

FIGURE 1. My health record [34]

Furthermore, by looking into the section labelled "Clinical Documents Uploaded", it can be easily determined that 898,011 have been uploaded. By dividing this number of docu 
ments by the 4,079,339 consumers registered, it is clear that there are only 0.22 documents for each consumer registered! There are several questions to consider: how many consumers are actively registered on the My Health Record system? Does each health provider upload new documents for a patient? The second set of statistics from the Australian Digital Health Agency website, released on 2 October 2016 (Figure 2), shows that the number of consumers registered by this date amounts to $4,218,970$. Comparing this with the consumers registered on 21 August (Figure 2), we find an increase of 139,631 new consumers registered in the period between 21 August and 2 October in the year 2016. This means that $3,247.23$ consumers registered each day during this period.

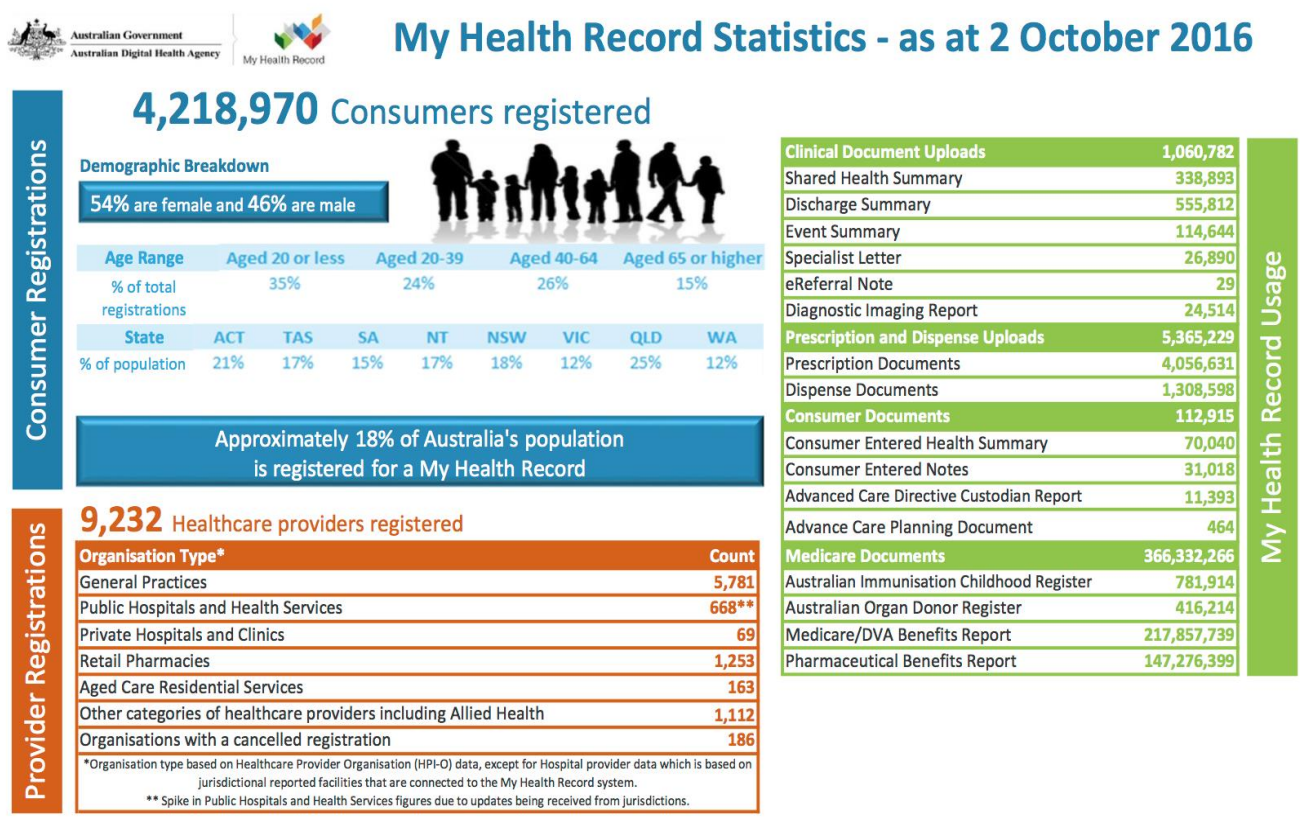

FIGURE 2 . My health record [34]

Significantly, the population of Australia amounts to 24,239,534 citizens [35, 36, 37, $38,39]$. According to the figures mentioned above, 20,020,564 citizens are not yet registered in the system, and, if the registration flow maintains a similar rate, it could take more than 15 years to achieve complete registration nationwide. However, this calculation entails the current population, without taking population growth into consideration.

\section{Enhancing Concerns of Losing Patient History Data}

The My Health Record website issues a warning to medical practitioners, cautioning against trusting their patients' history data as shown in the system:

"Clinical information you find within your patient's My Health Record should be interpreted in much the same way as other sources of health information. It is safest to assume the information in a patient's My Health Record is not a complete record of a patient's clinical history, so information should be verified from other sources and ideally, with the patient" [33]. This comment raises difficulties in encouraging citizens to continue using this system, and it will certainly increase related concerns. 


\section{CONCLUSION}

It can be concluded that the adoption of EHR systems has resulted in very effective and positive outcomes in many settings due to its implementation taking place all across the world. The first issue in the "My Health Record" system in Australia is the loss of patient's history data, which is one of the causes of the low registration numbers. In some cases in "My Health Record", there was a potential risk of modifying or deleting some data. Patients further have the option to opt out of the system. Additionally, there is a serious need to train not only healthcare practitioners and health staff on the use of the EHR system but also patients as there is a lack of participation in the system. Moreover, to ensure they understand the importance of the system's function. As EHR systems increase the efficiency of the work of medical professionals, there should be no fear of increased liability on the part of healthcare practitioners. In this paper, some relevant recommendations have been given in order to have a greater enrolling with Australian My Health Record system. The first recommendation is to shift from opt-out to opt-in approach, the second recommendation is to reduce risk by authentication, encryption, and improve user interface design. Also, there should be a separate system in each state and allow each state to have its own system to increase the efficiency. Last but not the least, a financial incentive will give My Health Record system a good promoter to use and upload patients' documents regally to the system by GPs. Lastly, rating GPs and clinics will increase their ambition to get a high level of rating, which will increase the usage of the system. Moreover, by accepting this paper's recommendations, it will be beneficial for all. There were limitations of this study. First, regarding My Health Record system status, all the statistic data were gathered from the internet such as My Health Record website and News websites. This limitation related to the stakeholders was caused by absence of permission to participate with stakeholders. Second, this study was designed to compare the global best practices of EHR with Australian My Health Record system. There was a need to visit another country to adopt their solutions like Sweden. Future research is needed to do experiments and surveys for knowing all the causes that gave rise to the lack of registration on My Health Record system.

\section{REFERENCES}

1. Ajami S, Bagheri-Tadi T. Barriers for adopting Electronic Health Records (EHRs) by physicians. Acta Informatica Medica. 2013; 21(2): 129-134. DOI: 10.5455/aim.2013.21.129-134

2. Health IT. What is an electronic health record (EHR)? 2016. Available from: goo.gl $/ \mathrm{j} 4 \mathrm{FdCH}$

3. Anderson JG. Social ethical and legal barriers to e-health. International Journal of Medical Informatics. 2007; 76(5): 480-483.

4. Nematollahi M, Abhari S, Garavand A. Attitudes and behaviors related to introduction of Electronic Health Record (EHR) among Shiraz University students in 2014. Journal of Health Management and Informatics. 2015; 2(3): 97-100.

5. Ludwick DA, Doucette J. Adopting electronic medical records in primary care: Lessons learned from health information systems implementation experience in seven countries. International Journal of Medical Informatics. 2009; 78(1): 22-31. DOI: 10.1016/j.ijmedinf.2008.06.005

6. Bracco D, Labeau F. Electronic health record: What do you expect from them? Critical Care Medicine. 2015; 43(6): 1342-1344. DOI: $10.1097 / \mathrm{ccm} .0000000000001007$ 
7. Best A, Greenhalgh T, Lewis S, Saul JE, Carroll S, Bitz J. Large-system transformation in health care: A realist review. The Milbank Quarterly. 2012; 90(3): 421-456. DOI: 10.1111/j.1468-0009.2012.00670.x

8. Häyrinen K, Saranto K, Nykänen P. Definition, structure, content, use and impacts of electronic health records: A review of the research literature. International Journal of Medical Informatics. 2008; 77(5): 291-304.

DOI: $10.1016 /$ j.ijmedinf.2007.09.001

9. Shortliffe EH, Cimino JJ, editors. Biomedical informatics: Computer applications in health care and biomedicine. New Jersy, NJ: Springer Science \& Business Media; 2013.

10. Hoyt RE, Bernstam EV, Johnson TR. Overview of medical Informatics. Practical Guide for the Healthcare Professional. 2013; 1(2): 123-127.

11. Juliansyah E, and Asnol UB. Workers behavior in maintaining health management waste in the final disposal. International Journal of Health and Medical Sciences. 2016; 2(2): 32-36. DOI: 10.20469/ijhms.2.30002-2

12. Prabsangob K. Relationships of health literacy diabetes knowledge and social support to self-care behavior among type 2 diabetic patients. International Journal of Health and Medical Sciences. 2016; 2(3): 68-72.

DOI: $10.20469 /$ ijhms.2.30005-3

13. Barnett G. History of the development of medical information systems at the Laboratory of Computer Science at Massachusetts General Hospital. Paper presetned at In A history of medical informatics 1990, Jun 1; Massachusetts, MA. DOI: /10.1145/41526.41531

14. Atherton J. Development of the electronic health record. Virtual Mentor. 2011; 13(3): 186-190.

15. Detmer DE, Steen EB, Dick RS, editors. The computer-based patient record: An essential technology for health care. New York, NY: National Academies Press; 1997.

16. Menachemi N, Collum TH. Benefits and drawbacks of electronic health record systems. Risk Management and Health care Policy. 2011; 4(3): 47-50. DOI: 10.2147/rmhp.s12985

17. Chen R, Klein G. The open EHR Java reference implementation project. Studies in Health Technology and Informatics. 2007; 129(1): 58-65. DOI: 10.1016/j.iccn.2013.12.002

18. Leslie H. Open EHR-the world's record. Pulse+ IT. 2007; 6(3): 50-55.

19. Glance D. New name and opt-out policy won't save the personal health record; 2015. Available from: goo.gl/UbKVVt

20. Falan S. EHR records in Sweden and what the future brings; 2016. Available from: goo.gl/JBWQ8C

21. Sungkhapong T, Prommete P, Martkoksoong N, Kittichottipanich B. "The health behaviors' modification for controlling and prevention of diabetes mellitus by using promise model at premruthai pravate community Bangkok. Journal of Advances in Health and Medical Sciences. 2016; 2(3): 97-101.

22. Wilson S. Why the Govt can't simply go opt-out for e-health; 2015. Available from: goo.gl/PLvN1a

23. Sinabutar R, Influence of the quality of medical and administrative services on the inpatients' loyalty at the Adventist Hospital Bandung. International Journal of Health and Medical Sciences. 2017; 3(1): 13-22.

24. Cowan P. Most Australian GP clinics aren't using e-health records. 2016; Available from: goo.gl/yktC1C

25. Heisey-Grove D, Patel V. Physician motivations for adoption of electronic health records; 2014. Available from: goo.gl/fssdVj

26. Bhagat S, Fontaine D, Gibson K. Danish healthcare information technology-an analytical study of consumer issues. Worcester, MA: Worcester Polytechnic Institute; 2010.

27. Gray BH, Bowden T, Johansen I, Koch S. Electronic health records: An international perspective on meaningful use. Issue Brief. 2011; 28(3): 1-8.

28. Pearce C, Bainbridge M. A personally controlled electronic health record for Australia. Journal of the American Medical Informatics Association. 2014; 21(4): 707-713.

29. Xu J, Gao X, Sorwar G, Croll P. Implementation of e-health record systems in Australia. The International Technology Management Review. 2013; 3(2): 92-104. DOI: 10.2991/itmr.2013.3.2.3

30. Lehnbom EC, McLachlan AJ, Jo-anne EB. A qualitative study of Australians' opinions about personally controlled electronic health records. Paper presented at the Health Informatics 2012, July 11; Sydney, AU. DOI: 10.1016/j.aucc.2011.04.004 
31. Budde P. Australia digital economy E-Health electronic health records; 2016. Available from: goo.gl/4HHfAK

32. Standing C, Cripps H. Critical success factors in the implementation of electronic health records: A two-case comparison. Systems Research and Behavioral Science. 2015; 32(1): 75-85. D0I: 10.1002/sres.2209

33. Horne M. Health on the hill: Political news from the nation's capital. Australian Medicine. 2017; 29(15): 25-29.

34. Everingham L. Coalition of National Nursing Organisations Report. Journal of Stomal Therapy Australia. 2011; 31(3): 24-30.

35. Ley S. Patients to get new myHealth Record: \$485m 'rescue' package to reboot Labor's e-health failures; 2015. Available from: goo.gl/gYjWBz

36. Ley S. My health record gets one million more reasons to sign up; 2016. Available from: goo.gl/Ym7DZg

37. My Health Record. Welcome to my health record; 2016. Available from: goo.gl/brwDcc

38. My Health Record. My Health record statistics; 2016. Available from: goo.gl/7Edvej

39. Australian Bureau of Statistics. Population clock; 2016. Available from: goo.gl/1dPXKY

— This article does not have any appendix. - 